\title{
Proving Two Partition Identities
}

R. DA SILVA, Centro de Matemática, Computação e Cognição, UFABC, 09210170 Santo André, SP, Brasil.

J.C. FILHO ${ }^{2}$ Departamento de Matemática e Computação, ICE - UNIFEI, 37500-903 Itajubá, MG, Brasil.

J.P.O. SANTOS, 3 Departamento de Matemática Aplicada, IMECC - UNICAMP, 13083-859 Campinas, SP, Brasil.

\begin{abstract}
In this paper we give combinatorial proofs for two partition identities. The first one solves a recent open question formulated by G. E. Andrews.
\end{abstract}

Keywords. Partition Identities, Combinatorics, Ferrers diagram.

\section{Introduction}

In [2] Andrews considers a variety of parity questions related to partition identities. We mention two of them just to illustrate results of this nature. The first one is a well known identity given by Euler.

Euler's Partition Identity. The number of partitions of any positive integer $n$ into distinct parts equals the number of partitions of $\mathrm{n}$ into odd parts.

In terms of generating functions: for $|\mathrm{q}|<1$,

$$
\prod_{j=1}^{n}\left(1+q^{j}\right)=\prod_{j=1}^{n} \frac{1}{\left(1-q^{2 j-1}\right)}
$$

The second one is due to B. Gordon ([6], [7]) and H. Göllnitz ([4], [5]) that independently introduced parity considerations as follows:

First Gollnitz-Gordon Identity. The number of partitions of $n$ into distinct non-consecutive parts with no even parts differing by exactly 2 equals the number of partitions of $\mathrm{n}$ into parts $\equiv 1,4$, or $7(\bmod 8)$.

At the end of [2], he presents a list of problems. In Section 2 we have a few definitions and the description of the Problem 5. In Section 3 we present our solution to the problem. In the last section we have a bijective proof for an identity related to the Durfee square of partitions.

\footnotetext{
${ }^{1}$ robson.dasilva@ufabc.edu.br

2jair@unifei.edu.br

3 josepli@ime.unicamp.br
} 


\section{Solving an Andrews's Problem}

It is important to mention that a solution for Problem 5 of Andrews's paper [2] was given in [8]. However, Yee's proof is based on some results related to generating functions for partitions and $q$-series. Our proof, although equivalent to Yee's one, deals with combinatorial aspects of the Ferrers diagram of a partition.

Before presenting the proof we recall a few definitions from [1] and [2].

Definition 2.1. A partition of a positive integer $n$ is a colection of positive integers $\lambda_{1}, \lambda_{2}, \ldots, \lambda_{s}$ such that $\lambda_{1} \geq \lambda_{2} \geq \cdots \geq \lambda_{s}$. Each $\lambda_{i}$ is called a part of the partition. We denote a partition by $\lambda_{1}+\lambda_{2}+\cdots+\lambda_{s}$ or $\left(\lambda_{1}, \lambda_{2}, \ldots, \lambda_{s}\right)$.

Example 2.1. The five partitions of 4 are: $4,3+1,2+2,2+1+1,1+1+1+1$.

Definition 2.2. A Ferrers diagram of a partition $\lambda_{1}+\lambda_{2}+\cdots+\lambda_{s}$ is an array of dots left justified having $\lambda_{1}$ dots in the first row, $\lambda_{2}$ dots in the second row and so on.

Definition 2.3. Given a partition, the largest possible square of dots, starting in the upper left-hand corner, contained in its Ferrers diagram is called the Durfee square of this partition.

Example 2.2. Below we have the Ferrers diagram of the partition $8+7+4+3$ with the Durfee square indicated.

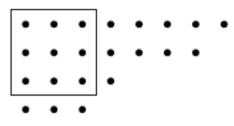

Figure 1: The Durfee square of a partition

Definition 2.4. Let $\lambda=\lambda_{1}+\lambda_{2}+\cdots+\lambda_{j}$ be a partition, where $\lambda_{1} \geq \lambda_{2} \geq \cdots \geq$ $\lambda_{j}$. The lower odd parity index of $\lambda, I_{l o}(\lambda)$, is defined as the maximum length of nondecreasing subsequences of $\left\{\lambda_{1}, \lambda_{2}, \ldots, \lambda_{j}\right\}$ whose terms alternate in parity starting with an odd $\lambda_{i}$.

Example 2.3.

$$
\begin{gathered}
I_{l o}(11+11+7+6+6+3+2+1+1)=5 \\
I_{l o}(14+11+8+6+6+3+3+2)=4
\end{gathered}
$$

In [2] Andrews asks for a combinatorial proof for

$$
\sum_{r, m \geq 0} p_{o}(r, m, N)(-1)^{r}= \begin{cases}1 & \text { if } N=n(3 n+1) / 2 \\ -1 & \text { if } N=n(3 n+5) / 2+1 \\ 0 & \text { otherwise }\end{cases}
$$

where $p_{o}(r, m, N)$ is the number of partitions of $N$ into $m$ distinct parts with lower odd parity index equal to $r$.

Consider, for example, the tables below. In the first columns we have partitions into distinct parts. The lower odd parity indices are shown in the second columns. 


\begin{tabular}{|l|c|}
\hline partitions of 11 & $I_{l o}$ \\
\hline \hline 11 & 1 \\
\hline $10+1$ & 2 \\
\hline $9+2$ & 1 \\
\hline $8+3$ & 2 \\
\hline $8+2+1$ & 2 \\
\hline $7+4$ & 1 \\
\hline $7+3+1$ & 1 \\
\hline $6+5$ & 2 \\
\hline $6+4+1$ & 2 \\
\hline $6+3+2$ & 2 \\
\hline $5+4+2$ & 1 \\
\hline $5+3+2+1$ & 3 \\
\hline
\end{tabular}

\begin{tabular}{|l|c|}
\hline partitions of 12 & $I_{l o}$ \\
\hline \hline 12 & 0 \\
\hline $11+1$ & 1 \\
\hline $10+2$ & 0 \\
\hline $9+3$ & 1 \\
\hline $9+2+1$ & 3 \\
\hline $8+4$ & 0 \\
\hline $8+3+1$ & 2 \\
\hline $7+5$ & 1 \\
\hline $7+4+1$ & 3 \\
\hline $7+3+2$ & 1 \\
\hline $6+5+1$ & 2 \\
\hline $6+4+2$ & 0 \\
\hline $6+3+2+1$ & 4 \\
\hline $5+4+3$ & 3 \\
\hline $5+4+2+1$ & 3 \\
\hline
\end{tabular}

From these tables it is easy to see that $\sum_{r, m \geq 0} p_{o}(r, m, 11)(-1)^{r}=0$ and $\sum_{r, m \geq 0} p_{o}(r, m, 12)(-1)^{r}=-1$

In order to present a combinatorial proof of (2.2), we identify each partition with its Ferrers diagram, the standard graphical representation of a partition.

The combinatorial proof we are going to show is based on the one-to-one correspondence found by F. Franklin in 1881 to prove Legendre's combinatorial version of Euler's pentagonal number theorem (see [1]).

\section{The Combinatorial Proof}

Let $D_{e}(N)$ (resp. $D_{o}(N)$ ) be the number of partitions of $N$ into distinct parts having even (resp. odd) lower odd parity index. Then, we can rephrase (2.2) in the following theorem.

Theorem 3.1.

$$
D_{e}(N)-D_{o}(N)= \begin{cases}1 & \text { if } N=n(3 n+1) / 2 \\ -1 & \text { if } N=n(3 n+5) / 2+1 \\ 0 & \text { otherwise }\end{cases}
$$

Proof. We shall establish a one-to-one correspondence between the partitions enumerated by $D_{e}(N)$ and those enumerated by $D_{o}(N)$. This correspondence will not work for some partitions of the numbers of the form $N=n(3 n+1) / 2$ and $N=n(3 n+5) / 2+1$.

Each partition $\lambda=\lambda_{1}+\lambda_{2}+\cdots+\lambda_{s}$ of $N$ into distinct parts has a smallest part $\lambda_{s}$ and the largest part $\lambda_{1}$ is the first element of a decreasing sequence of $l$ consecutive integers that are parts of $\lambda$. For example, below we have the Ferrers diagrams of the partitions $8+7+4+3$ and $8+7+6+3+2$ with their parameter $\lambda_{s}$ and $l$. 

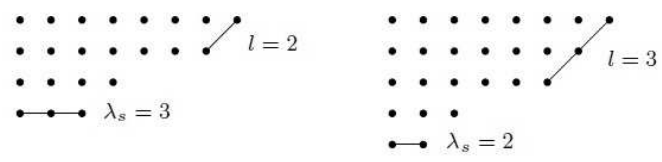

Figure 2: Two partitions and their parameter $\lambda_{s}$ and $l$

In order to establish the one-to-one correspondence we consider the following two cases.

Case 1: $\lambda_{s}>l$. In this case, we remove 1 from the parts $\lambda_{1}, \lambda_{2}, \ldots, \lambda_{l}$ and form a new smallest part of size $l$. For example,

$$
8+7+4+3 \longmapsto 7+6+4+3+2,
$$

or, in terms of the graphical representation,

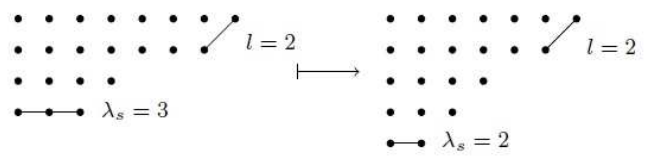

Figure 3: A partition of Case 1

Case 2: $\lambda_{s} \leq l$. In this case, we remove the smallest part and add 1 to the first $\lambda_{s}$ of the $l$ largest parts. For example,

$$
8+7+6+3+2 \longmapsto 9+8+6+3
$$

that is,

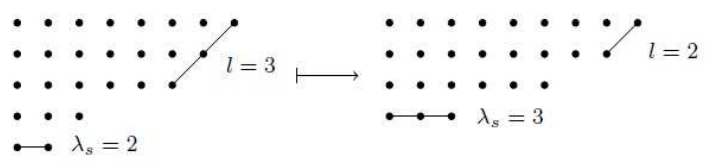

Figure 4: A partition of Case 2

Note that exactly one case is applicable to a given partition into distinct parts. Then, it seems that the mapping establishes a one-to-one correspondence. This is true except for certain partitions like $5+4+3$.

The procedure described in Case 1 is not applicable to those partitions having $l$ parts and $\lambda_{s}=l+1$, in which case the number being partitioned is

$$
(l+1)+(l+2)+\cdots+(2 l)=\frac{l(3 l+1)}{2} .
$$


While the procedure described in Case 2 is not applicable to those partitions having $l$ parts and $\lambda_{s}=l$, in which case the number being partitioned is

$$
l+(l+1)+\cdots+(2 l-1)=\frac{l(3 l-1)}{2}=\frac{m(3 m+5)}{2}+1,
$$

where $m=l-1$. For example, Case 1 and Case 2 are not applicable to

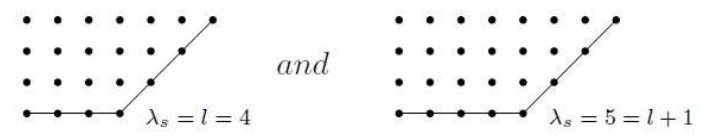

Figure 5: Partitions that are neither of Case 1 nor of Case 2

Note that partitions of the form $\lambda=(2 l-1)+\cdots+(l+1)+l$ have an odd lower odd parity index: if $l$ is odd, then $I_{l o}(\lambda)=l$; if $l$ is even, then $I_{l o}(\lambda)=l-1$. We also note that partitions of the form $\lambda=2 l+\cdots+(l+2)+(l+1)$ have an even lower odd parity index: if $l$ is odd, then $I_{l o}(\lambda)=l-1$; if $l$ is even, then $I_{l o}(\lambda)=l$.

In order to finish the proof we shall show that the correspondence described above changes the parity of the index $I_{l o}(\lambda)$, when $\lambda=\lambda_{1}+\lambda_{2}+\cdots+\lambda_{s}$ is neither a partition into $l$ parts with $\lambda_{s}=l$ nor a partition into $l$ parts with $\lambda_{s}=l+1$. Again, we consider the two cases above.

We will call $\mu$ the image partition obtained from $\lambda=\lambda_{1}+\lambda_{2}+\cdots+\lambda_{l}+\lambda_{l+1}+$ $\cdots+\lambda_{s}$ by the correspondence above, i.e.,

$$
\mu=\left\{\begin{array}{l}
\left(\lambda_{1}-1\right)+\cdots+\left(\lambda_{l}-1\right)+\lambda_{l+1}+\cdots+\lambda_{s}+l, \text { if } \lambda_{s}>l, \\
\left(\lambda_{1}+1\right)+\cdots+\left(\lambda_{\lambda_{s}}+1\right)+\lambda_{\lambda_{s}+1}+\cdots+\lambda_{l}+\cdots+\lambda_{s-1}, \text { if } \lambda_{s} \leq l .
\end{array}\right.
$$

We shall show that $I_{l o}(\lambda)$ and $I_{l o}(\mu)$ have different parities.

Case 1: $\lambda_{s}>l$. We split this case into two sub-cases.

$\underline{\text { Sub-case 1.1: }} s=l$. In this sub-case, $\lambda=\lambda_{1}+\lambda_{2}+\cdots+\lambda_{l}$ and $\mu=\left(\lambda_{1}-1\right)+$ $\left(\lambda_{2}-1\right)+\cdots+\left(\lambda_{l}-1\right)+l$. Hence, if $I_{l o}(\lambda)=l$, then $\lambda_{l}$ is odd and, consequently,

- if $l$ is odd, then $I_{l o}(\mu)=l+1$.

- if $l$ is even, then $I_{l o}(\mu)=l-1$. 
If $I_{l o}(\lambda)=l-1$, then $\lambda_{l}$ is even and, consequently,

- if $l$ is odd, then $I_{l o}(\mu)=l$.

- if $l$ is even, then $I_{l o}(\mu)=l$.

For example

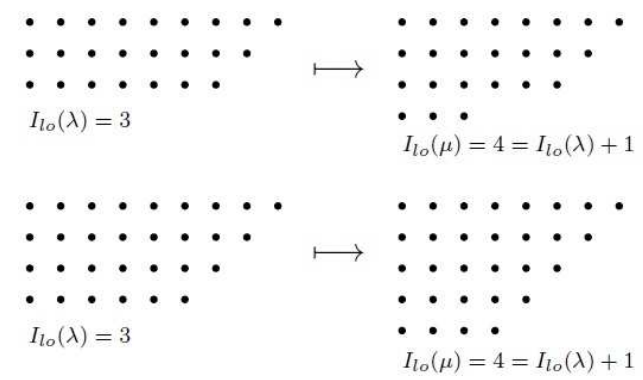

Figure 6: Examples of change of parity in Sub-case 1.1

Sub-case 1.2: $s>l$. The parts $\lambda_{1}, \lambda_{2}, \ldots, \lambda_{l}$ of $\lambda$ alternate in parity. The same is true for the parts $\left(\lambda_{1}-1\right),\left(\lambda_{2}-1\right), \ldots,\left(\lambda_{l}-1\right)$ of $\mu$. Then in order to determine the index $I_{l o}(\mu)$ in terms of $I_{l o}(\lambda)$ we have to look at the parity of $\lambda_{l}$ and $\lambda_{l+1}$ as well as the parity of $l$ and $\lambda_{s}$. While the parity of $\lambda_{l}$ is changed by the correspondence, the same does not happen with $\lambda_{l+1}$. For example,

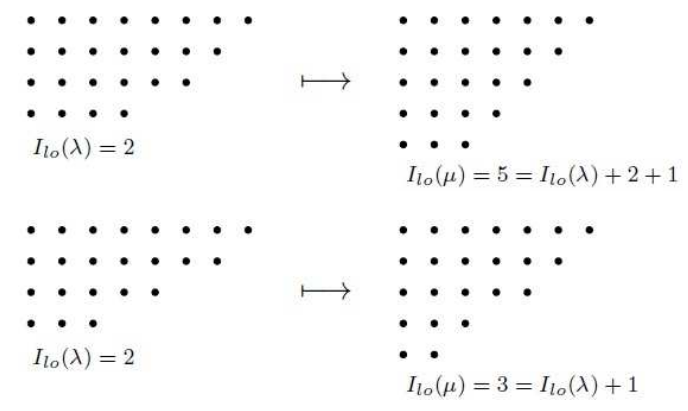

Figure 7: Examples of change of parity in Sub-case 1.2

As there are two choices for the parities of $l, \lambda_{l}, \lambda_{l+1}$, and $\lambda_{s}$, then we have to verify, in this sub-case, all the $2^{4}$ possibilities. In the table below we summarise the results. In this table $e$ means even and $o$ means odd. 


\begin{tabular}{|c|c|c|c|c|}
\hline$l$ & $\lambda_{l}$ & $\lambda_{l+1}$ & $\lambda_{s}$ & $I_{l o}(\mu)$ \\
\hline \hline $\mathrm{e}$ & $\mathrm{e}$ & $\mathrm{e}$ & $\mathrm{e}$ & $I_{l o}(\lambda)+1$ \\
\hline $\mathrm{e}$ & $\mathrm{e}$ & $\mathrm{e}$ & $\mathrm{o}$ & $I_{l o}(\lambda)+1$ \\
\hline $\mathrm{e}$ & $\mathrm{e}$ & $\mathrm{o}$ & $\mathrm{e}$ & $I_{l o}(\lambda)-1$ \\
\hline $\mathrm{e}$ & $\mathrm{e}$ & $\mathrm{o}$ & $\mathrm{o}$ & $I_{l o}(\lambda)-1$ \\
\hline $\mathrm{e}$ & $\mathrm{o}$ & $\mathrm{e}$ & $\mathrm{e}$ & $I_{l o}(\lambda)-1$ \\
\hline $\mathrm{e}$ & $\mathrm{o}$ & $\mathrm{e}$ & $\mathrm{o}$ & $I_{l o}(\lambda)-1$ \\
\hline $\mathrm{e}$ & $\mathrm{o}$ & $\mathrm{o}$ & $\mathrm{e}$ & $I_{l o}(\lambda)+1$ \\
\hline $\mathrm{e}$ & $\mathrm{o}$ & $\mathrm{o}$ & $\mathrm{o}$ & $I_{l o}(\lambda)+1$ \\
\hline $\mathrm{o}$ & $\mathrm{e}$ & $\mathrm{e}$ & $\mathrm{e}$ & $I_{l o}(\lambda)+3$ \\
\hline $\mathrm{o}$ & $\mathrm{e}$ & $\mathrm{e}$ & $\mathrm{o}$ & $I_{l o}(\lambda)+1$ \\
\hline $\mathrm{o}$ & $\mathrm{e}$ & $\mathrm{o}$ & $\mathrm{e}$ & $I_{l o}(\lambda)+1$ \\
\hline $\mathrm{o}$ & $\mathrm{e}$ & $\mathrm{o}$ & $\mathrm{o}$ & $I_{l o}(\lambda)-1$ \\
\hline $\mathrm{o}$ & $\mathrm{o}$ & $\mathrm{e}$ & $\mathrm{e}$ & $I_{l o}(\lambda)+1$ \\
\hline $\mathrm{o}$ & $\mathrm{o}$ & $\mathrm{e}$ & $\mathrm{o}$ & $I_{l o}(\lambda)-1$ \\
\hline $\mathrm{o}$ & $\mathrm{o}$ & $\mathrm{o}$ & $\mathrm{e}$ & $I_{l o}(\lambda)+3$ \\
\hline $\mathrm{o}$ & $\mathrm{o}$ & $\mathrm{o}$ & $\mathrm{o}$ & $I_{l o}(\lambda)+1$ \\
\hline
\end{tabular}

Table 1: The 16 possibilities of Sub-case 1.2

Case 2: $\lambda_{s} \leq l$. Again we consider two sub-cases.

Sub-case 2.1: $\lambda_{s}<l$. In this case the operation of removing the smallest part of

$$
\lambda=\lambda_{1}+\cdots+\lambda_{\lambda_{s}}+\lambda_{\lambda_{s}+1}+\cdots+\lambda_{l}+\lambda_{l+1}+\cdots+\lambda_{s-1}+\lambda_{s}
$$

and adding 1 to each of its first $\lambda_{s}$ largest parts produces

$$
\mu=\left(\lambda_{1}+1\right)+\cdots+\left(\lambda_{\lambda_{s}}+1\right)+\lambda_{\lambda_{s}+1}+\cdots+\lambda_{l}+\lambda_{l+1}+\cdots+\lambda_{s-1} .
$$

Since $\lambda_{\lambda_{s}}$ and $\lambda_{\lambda_{s}+1}$ have opposite parities, we have to consider the parities of $\lambda_{s}, \lambda_{s-1}$, and $\lambda_{\lambda_{s}}$. For example, by this operation we can obtain

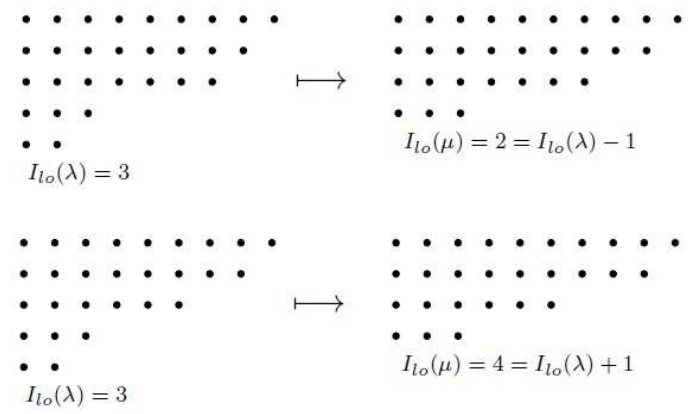

Figure 8: Examples of change of parity in Sub-case 2.1

The table below shows the $2^{3}$ possibilities and the values of $I_{l o}(\mu)$ in terms of $I_{l o}(\lambda)$. 


\begin{tabular}{|c|c|c|c|}
\hline$\lambda_{s}$ & $\lambda_{s-1}$ & $\lambda_{\lambda_{s}}$ & $I_{l o}(\mu)$ \\
\hline \hline $\mathrm{e}$ & $\mathrm{e}$ & $\mathrm{e}$ & $I_{l o}(\lambda)-1$ \\
\hline $\mathrm{e}$ & $\mathrm{e}$ & $\mathrm{o}$ & $I_{l o}(\lambda)-1$ \\
\hline $\mathrm{e}$ & $\mathrm{o}$ & $\mathrm{e}$ & $I_{l o}(\lambda)-1$ \\
\hline $\mathrm{e}$ & $\mathrm{o}$ & $\mathrm{o}$ & $I_{l o}(\lambda)-1$ \\
\hline $\mathrm{o}$ & $\mathrm{e}$ & $\mathrm{e}$ & $I_{l o}(\lambda)-3$ \\
\hline $\mathrm{o}$ & $\mathrm{e}$ & $\mathrm{o}$ & $I_{l o}(\lambda)-3$ \\
\hline $\mathrm{o}$ & $\mathrm{o}$ & $\mathrm{e}$ & $I_{l o}(\lambda)-1$ \\
\hline $\mathrm{o}$ & $\mathrm{O}$ & $\mathrm{o}$ & $I_{l o}(\lambda)-1$ \\
\hline
\end{tabular}

Table 2: The 8 possibilities of Sub-case 2.1

Sub-case 2.2: $\lambda_{s}=l$. In this sub-case we have to look at the parity of $\lambda_{s}, \lambda_{s-1}, \lambda_{l}$, and $\lambda_{l+1}$. For instance, if $\lambda_{s}$ and $\lambda_{s-1}$ are odd and both $\lambda_{l}$ and $\lambda_{l+1}$ are even, then $I_{l o}(\mu)=I_{l o}(\lambda)+1$. We summarize all possibilities in the next table.

\begin{tabular}{|c|c|c|c|c|}
\hline$\lambda_{s}$ & $\lambda_{s-1}$ & $\lambda_{l}$ & $\lambda_{l+1}$ & $I_{l o}(\mu)$ \\
\hline \hline $\mathrm{e}$ & $\mathrm{e}$ & $\mathrm{e}$ & $\mathrm{e}$ & $I_{l o}(\lambda)+1$ \\
\hline $\mathrm{e}$ & $\mathrm{e}$ & $\mathrm{e}$ & $\mathrm{o}$ & $I_{l o}(\lambda)-1$ \\
\hline $\mathrm{e}$ & $\mathrm{e}$ & $\mathrm{o}$ & $\mathrm{e}$ & $I_{l o}(\lambda)-1$ \\
\hline $\mathrm{e}$ & $\mathrm{e}$ & $\mathrm{o}$ & $\mathrm{o}$ & $I_{l o}(\lambda)+1$ \\
\hline $\mathrm{e}$ & $\mathrm{o}$ & $\mathrm{e}$ & $\mathrm{e}$ & $I_{l o}(\lambda)+1$ \\
\hline $\mathrm{e}$ & $\mathrm{o}$ & $\mathrm{e}$ & $\mathrm{o}$ & $I_{l o}(\lambda)-1$ \\
\hline $\mathrm{e}$ & $\mathrm{o}$ & $\mathrm{o}$ & $\mathrm{e}$ & $I_{l o}(\lambda)-1$ \\
\hline $\mathrm{e}$ & $\mathrm{o}$ & $\mathrm{o}$ & $\mathrm{o}$ & $I_{l o}(\lambda)+1$ \\
\hline $\mathrm{o}$ & $\mathrm{e}$ & $\mathrm{e}$ & $\mathrm{e}$ & $I_{l o}(\lambda)-1$ \\
\hline $\mathrm{o}$ & $\mathrm{e}$ & $\mathrm{e}$ & $\mathrm{o}$ & $I_{l o}(\lambda)-3$ \\
\hline $\mathrm{o}$ & $\mathrm{e}$ & $\mathrm{o}$ & $\mathrm{e}$ & $I_{l o}(\lambda)-3$ \\
\hline $\mathrm{o}$ & $\mathrm{e}$ & $\mathrm{o}$ & $\mathrm{o}$ & $I_{l o}(\lambda)-1$ \\
\hline $\mathrm{o}$ & $\mathrm{o}$ & $\mathrm{e}$ & $\mathrm{e}$ & $I_{l o}(\lambda)+1$ \\
\hline $\mathrm{o}$ & $\mathrm{o}$ & $\mathrm{e}$ & $\mathrm{o}$ & $I_{l o}(\lambda)-1$ \\
\hline $\mathrm{o}$ & $\mathrm{o}$ & $\mathrm{o}$ & $\mathrm{e}$ & $I_{l o}(\lambda)-1$ \\
\hline $\mathrm{o}$ & $\mathrm{o}$ & $\mathrm{o}$ & $\mathrm{o}$ & $I_{l o}(\lambda)+1$ \\
\hline
\end{tabular}

Table 3: The 16 possibilities of Sub-case 2.2

As one can see from the tables above the parity of the index $I_{l o}$ is always changed by the one-to-one correspondence described above. Therefore the theorem is proved.

\section{The Second Bijection}

In this section we present a bijective proof for the partition identity below, which is related to the Durfee square (the largest possible square contained within the 
Ferrers diagram of a partition starting in the upper left-hand corner). This identity comes from two different interpretations for the Fibonacci Numbers (see [3]).

Theorem 4.2. The number of partitions into at most $n$ parts without gaps ${ }^{4}$ and having each part appearing at least twice is equal to the number of partitions where the largest part is the side of its Durfee square and the largest part plus the number of parts is less than or equal to $n$.

Proof. Let $n$ be a non-negative integer. Define $\mathcal{A}$ as the set of partitions into at most $n$ parts without gaps and having each part appearing at least twice. We also define $\mathcal{B}$ as being the set of partitions where the largest part is the side of its Durfee square and the largest part plus the number of parts is less than or equal to $n$.

Note that the empty partition is in both sets $\mathcal{A}$ and $\mathcal{B}$, consequently we associate these two partitions. Now we describe the bijection between $\mathcal{A}$ and $\mathcal{B}$ for non-empty partitions. In order to do this we associate each partition with its Ferrers diagrams. Given a non-empty partition $\lambda \in \mathcal{A}$, with largest part $p$, we obtain a partition $\mu \in \mathcal{B}$, with Durfee square of side $p$, by the following procedure:

- delete $p$ dots from the first column of $\lambda$

- move $p-2, p-4, \ldots, p-2 \times\left\lfloor\frac{p}{2}\right\rfloor$ dots from the columns $2,3, \ldots,\left\lfloor\frac{p}{2}\right\rfloor+1$ to the columns $p, p-1, p-2, \ldots, p-\left\lfloor\frac{p}{2}\right\rfloor+1$, respectively.

For example, consider the partition $\lambda=7+7+6+6+6+6+5+5+4+4+$ $4+3+3+3+3+2+2+2+1+1+1$. The partition $\mu$ obtained by the above procedure is $\mu=7+7+7+7+7+7+7+6+6+4+3+3+2+1$ or, in terms of Ferrers diagrams,

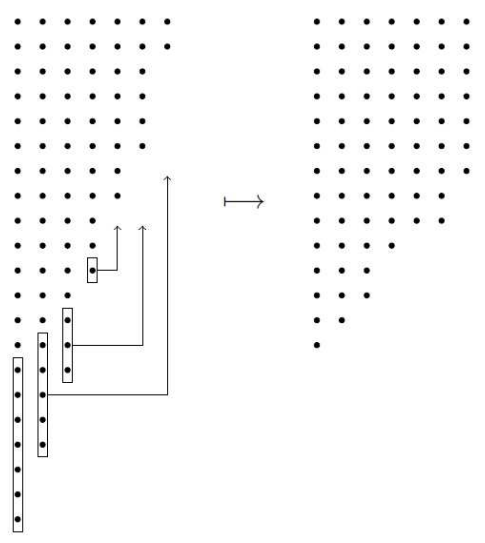

Figure 9: An example of a partition of $\mathcal{A}$ and its corresponding partition of $\mathcal{B}$

\footnotetext{
${ }^{4}$ all positive integer less than or equal to the largest part are parts of the partition
} 
It is important to note that the resulting diagram still represents a partition. This is ensured by the condition that each part appears at least twice.

Given a partition $\sigma \in \mathcal{B}$ we obtain a partition $\delta \in \mathcal{A}$ just by inverting the above procedure. As an example, consider $\sigma=6+6+6+6+6+6++3+1$; the partition associated to $\sigma$ is $\delta=6+6+5+5+4+4+3+3+3+2+2+1+1+1$ :

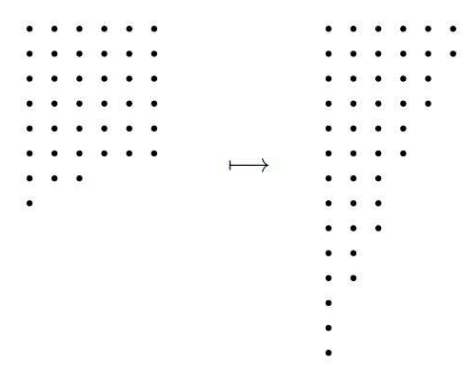

Figure 10: An example of a partition of $\mathcal{B}$ and its corresponding partition of $\mathcal{A}$

Resumo. Neste artigo fornecemos provas combinatórias para duas identidades de partições. A primeira resolve uma questão recentemente formulada por G. E. Andrews.

Palavras-chave. Identidades de partições, Combinatória, Diagrama de Ferrers.

\section{References}

[1] G.E. Andrews, "The Theory of Partitions", Cambridge University Press, 1984.

[2] G.E. Andrews, Parity in partition identities, The Ramanujan Journal, 23 (2010), 45-90.

[3] J.C. Filho, "Variações do Diagrama de Ferrers, Partições Planas e Funções Geradoras", Tese de Doutorado, IMECC, UNICAMP, Campinas, SP, 2006.

[4] H. Göllnitz, Einfache Partionen, Diplomarbeit W.S. 1960, Göttingen, 65 pp.

[5] H. Göllnitz, Partitionen mit Differenzenbedingungen, J. Reine Angew. Math, 225 (1967), 154-190.

[6] B. Gordon, Some Ramanujan-like continued fractions, Abstracts of Short Communications, Int. Congr. of Math., 29-30, Stockholm, 1962.

[7] B. Gordon, Some continued fractions of the Rogers-Ramanujan type, Duke Math. J., 31 (1965), 741-748.

[8] A.J. Yee, Ramanujan's partial theta series and parity in partitions, The $R a$ manujan Journal, 23 (2010), 215-225. 